\title{
A New Regularized Solution to Ill-Posed Problem in Coordinate Transformation
}

\author{
Xuming $\mathrm{Ge}^{1}$, Jicang $\mathrm{Wu}^{1,2}$ \\ ${ }^{1}$ Department of Surveying and Geo-Informatics, Tongji University, Shanghai, China \\ ${ }^{2}$ Key Laboratory of Advanced Surveying Engineering of State Bureau of Surveying and Mapping, Shanghai, China \\ Email: gexumingxmy@163.com,jcwu@tongji.edu.cn
}

Received July 23, 2011; revised September 21, 2011; accepted November 15, 2011

\begin{abstract}
Coordinates transformation is generally required in GPS applications. If the transformation parameters are solved with the known coordinates in a small area using the Bursa model, the precision of transformed coordinates is generally very poor. Since the translation parameters and rotation parameters are highly correlated in this case, a very large condition number of the coefficient matrix $\boldsymbol{A}$ exists in the linear system of equations $\boldsymbol{A} x \approx b$. Regularization is required to reduce the effects caused by the intrinsic ill-conditioning of the problem and noises in the data, and to stabilize the solution. Based on advanced regularized methods, we propose a new regularized solution to the ill-posed coordinate transformation problem. Simulation numerical experiments of coordinate transformation are given to shed light on the relationship among different regularization approaches. The results indicate that the proposed new method can obtain a more reasonable resolution with higher precision and/or robustness.
\end{abstract}

Keywords: Bursa; Coordinate Transformation; Ill-Posed; Methodology

\section{Introduction}

Coordinate transformation plays a very importance role in the numerical treatment of global positioning system. For transforming GPS coordinates from WGS-84 coordinate system to a local coordinate system, the Bursa model is generally used to solve transformation parameters, including three translation parameters, three rotation parameters and one scale parameter. From a theoretical point of view, a great number of algorithms have been developed to solve these problems. Early publications such as Grafarend et al. [1], Vanicek and Steeves [2], Vanicek et al. [3], Yang [4], and Grafarend and Awange [5] have given some detail algorithms of coordinate transformation. In general, their algorithms to solve transformation parameters are all based on the classical least squares criterion. Recently, better methods are available in literature, e.g. hard or soft fixing of certain transformation parameters (e.g. rotation around some coordinate axes are strongly correlated with translations), reduction of coordinates to the centre of "gravity" etc., however, ill-posed problems were rarely considered in those methods. Actually, illposed problems often impact this kind of data processing.

In the coordinate transformation, the Bursa model is more suitable for global data, so global distributed data is normally required to solve coordinate transformation parameters. However, an engineering GPS control network covers only hundreds of square kilometers, or even smaller. In this case, the translation and rotation parameters are highly correlated and the mathematical model is thus ill-posed. Generally, parameters obtained by traditional algorithms from the ill-posed model will have poor precision and robustness.

As is well known, regularization is a powerful tool to solve ill-posed problems, which have been widely applied to solve inverse ill-posed geodetic problems and signal processing. Tikhonov proposed a well-known regularization method to ill-posed models [6,7]. Golub [8] proposed a singular value decomposition $(S V D)$ method to least squares solutions and Hansen analyzed the truncated $S V D$ as a method for regularization [9] in mathematics. $\mathrm{Xu}$ and Rummel [10] advanced a multiple parameters regularization method to solve ill-posed problems in geodesy [11]. These investigations are almost based on pure mathematics and not considered the characteristics of practical surveying applications.

In this paper, we will propose a new approach to solve ill-posed problems in the coordinate transformation. The process starts with discussion of early methods for solving ill-posed problems in mathematics, and analysis of the characteristics of ill-posed coordinate transformation problems. Taking account of the characteristics of coordinate transformation in GPS applications, a new algo- 
rithm is proposed. On other hand, based on the new algorithm we formulate a new approach to choose regularization parameters. In the new algorithm, information borne by small and large singular values has been kept by different methods.

\section{Main Equations and Notations}

We introduce main equations and notations used throughout this paper. Matrices are represented by the uppercase English alphabet and $\boldsymbol{I}$ denotes identity matrix. Scalars are represented by lowercase Greek letters or English alphabet with double subscripts. Superscript $T$ denotes the transpose of a matrix. Let $\|A\|_{2}$ denotes the 2-norm of matrix $\boldsymbol{A}$. Let $\mathfrak{R}(A)$ denotes the range of the matrix $\boldsymbol{A}$. In this paper, we deal with the linear finite-dimension equations.

$$
\boldsymbol{A} x \approx b, \boldsymbol{A} \in \mathfrak{R}^{m \times n}, b \in \mathfrak{R}^{m \times d}, m>n, m>d
$$

When the rotations are small, the mathematical model of the Bursa model can recast as

$$
\begin{aligned}
& {\left[\begin{array}{lllllll}
1 & 0 & 0 & 0 & -z^{\prime} & y^{\prime} & x^{\prime} \\
0 & 1 & 0 & z^{\prime} & 0 & -x^{\prime} & y^{\prime} \\
0 & 0 & 1 & -y^{\prime} & x^{\prime} & 0 & z^{\prime}
\end{array}\right]_{i}\left[\begin{array}{l}
T \\
R \\
\mu
\end{array}\right]} \\
& =\left[\begin{array}{l}
x \\
y \\
z
\end{array}\right]-\left[\begin{array}{l}
x^{\prime} \\
y^{\prime} \\
z^{\prime}
\end{array}\right]
\end{aligned}
$$

where $T=\left[\begin{array}{lll}x_{0} & y_{0} & z_{0}\end{array}\right]^{T}, R=\left[\begin{array}{lll}\varepsilon_{x} & \varepsilon_{y} & \varepsilon_{z}\end{array}\right]^{T}$ and $\mu$ denote the translate parameters, rotation parameters and scale parameter, respectively.

We also denote the SVD of $\boldsymbol{A}$ by

$$
\boldsymbol{A}=U \sum V^{T}=\sum_{i=1}^{n} u_{i} \sigma_{i} v_{i}^{T}
$$

with

$$
\begin{gathered}
U=\left[u_{1}, u_{2}, \cdots, u_{n}\right], u_{i} \in \mathfrak{R}^{m} \quad U^{T} U=I_{n}, \\
V=\left[v_{1}, v_{2}, \cdots, v_{n}\right], v_{i} \in \mathfrak{R}^{n}, \quad V^{T} V=I_{n},
\end{gathered}
$$

and

$$
\begin{gathered}
\sum=\operatorname{diag}\left(\sigma_{1}, \sigma_{2}, \cdots, \sigma_{n}\right) \in \mathfrak{R}^{n \times n}, \\
\sigma_{1} \geq \sigma_{2} \geq \cdots \geq \sigma_{n} \geq 0 .
\end{gathered}
$$

The numbers $\sigma_{i}$ are called the singular values of $\boldsymbol{A}$, while the vectors $\boldsymbol{u}_{i}$ and $\boldsymbol{v}_{i}$ are the left and right singular vectors of $\boldsymbol{A}$, respectively.

\section{Methodologies for Ill-Posed Problem}

The ill-posed problems are with a very large condition number of the coefficient matrix $\boldsymbol{A}$ in a linear system as Equation (1), and most of discussion about solutions of ill-condition-ed matrices require knowledge of the SVD of the matrix $\boldsymbol{A}$ [12]. In particular, the condition number of $\boldsymbol{A}$ is defined as the ratio between the largest and the smallest singular values of $\boldsymbol{A}$.

The numerical treatment of equations with an ill-conditioned coefficient matrix depends on the type of ill-condition of $\boldsymbol{A}$. There are two classes of ill-posed problems. The first is rank-deficient problems in which the matrix A has a cluster of small singular values, and there is a well-determined gap between large and small singular values. The second is the discrete ill-posed problem that all of the singular values of $\boldsymbol{A}$, on the average, decay gradually to zero, that means there is no gap in the singular value spectrum. For some more details on ill-posed problems, the reader may refer to Hansen [13] and Tarantola [14].

Considering an ill-posed linear system as Equation (1), with nonsingular matrix $\boldsymbol{A}$ and the right-hand side b polluted by white noise, thus Equation (1) can be rewritten in the form

$$
\boldsymbol{A} x \approx \boldsymbol{b}, \boldsymbol{A} \in \mathfrak{R}^{m \times n}, \boldsymbol{b} \in \mathfrak{R}^{m \times d}, \boldsymbol{b}=b_{\text {exact }}+b_{\text {noise }}
$$

where

$$
\left\|b_{\text {exact }}\right\|_{2} \gg\left\|b_{\text {noise }}\right\|_{2}
$$

We wish to approximate

$$
x_{\text {exact }}=A^{-1} b_{\text {exact }}
$$

However, $b_{\text {exact }}$ cannot be directly divided from a observation vector $\boldsymbol{b}$, we get the direct least squares solution

$$
x_{L S}=\left(A^{T} \boldsymbol{A}\right)^{-1} A^{T} \boldsymbol{b}
$$

It is well known that the direct solution is completely dominated by noise, when the coefficient matrix $\boldsymbol{A}$ is ill-posed. Based on the SVD of $\boldsymbol{A}$, the direct solution can recast

$$
\begin{gathered}
x_{\text {naive }}=\sum_{i=1}^{n} \frac{u_{i}^{T} \boldsymbol{b}}{\sigma_{i}} v_{i} \\
=\left(\sum_{i=1}^{n} \frac{u_{i}^{T} b_{\text {exact }}}{\sigma_{i}} v_{i}\right)+\left(\sum_{i=1}^{n} \frac{u_{i}^{T} b_{\text {noise }}}{\sigma_{i}} v_{i}\right)
\end{gathered}
$$

Considering Equation (8), to get an applicable directly solution, it is necessary to make sure that the following assumption which is called the Discrete Picard Condition is satisfied: The exact SVD coefficient $\left|u_{i}^{T} \boldsymbol{b}\right|$ decay faster than the $\sigma_{i}$. More details and analysis can also be fund in Hansen $[13,15]$. When the coefficient matrix $\boldsymbol{A}$ is ill-posed, the solution $x_{\text {naive }}$ is with poor robustness and not applicable.

Next considering Equation (9), the first sum in Equation (9) must converge to $x_{\text {exact }}$. It means the numerators in the first sum in Equation (9) decay faster than (or, at 
least, as fast as) the singular values in denominators, with growing $i$. On other hand, $b_{\text {noise }}$ represents white noise in second sum in Equation (9). It means noise Fourier coefficients $\left|u_{i}^{T} b_{\text {noise }}\right|$ cannot decay faster than (or, at least, after some point) the singular values, with growing $i$ in ill-posed problems. That means $b_{\text {noise }}$ will dominate the solution $x_{\text {naive }}$ after some point. Consequently, ill-posed problems with perturbations in observation vector may magnify the noise information by the corresponding singular value which is very small. The magnified noise completely cover the useful information, $\left\|A^{-1} b_{\text {exact }}\right\| \leq\left\|A^{-1} b_{\text {noise }}\right\|$, and $x_{\text {naive }}$ thus does not ap-
proximate to $x_{\text {exact }}$.

For solving the first class ill-posed problems, for which there is a well-determined gap between the large and small singular value of coefficient matrix $\boldsymbol{A}$, Hansen proposed a well-known approach [9,13,16,] - truncated SVD. The key idea in this approach is to obtain the truncated point $k$, then Equation (8) can recast as

$$
x_{k}=\sum_{i=1}^{k} \frac{u_{i}^{T} b}{\sigma_{i}} v_{i}
$$

The solution $x_{k}$ is referred to as the truncated SVD solution.

For solving the second class ill-posed problems in which the singular values of matrix $\boldsymbol{A}$ decay gradually to zero, the above TSVD method cannot applied for solving this problem. Tikhonov regularization method is a wellknown method for solving this kind of ill-posed problem. The key idea in Tikhonov's method is to incorporate a priori assumptions about the size and smoothness of the desired solution. Tikhonov regularization in general form leads to the minimization problem

$$
\min \left\{\|A x-b\|_{2}^{2}+\lambda^{2}\|L x\|_{2}^{2}\right\}
$$

where the regularization parameter $\lambda$ controls the weight given to minimization of the regularization term, relative to the minimization of the residual norm, and $L$ represents regularization matrix. In this paper, we only concern $L=I$. Then the Tikhonov solution $x_{\lambda}$ is given by

$$
x_{\lambda}=\left(A^{T} A+\lambda^{2} I\right)^{-1} A^{T} b
$$

Tikhonov method can be rewritten in SVD form with filter factor defined as

$$
f_{i}=\frac{\sigma_{i}^{2}}{\sigma_{i}^{2}+\lambda^{2}}
$$

and the regularized solution is

$$
x_{\text {reg }}=\sum_{i=1}^{\operatorname{rank}(A)} f_{i} \frac{u_{i}^{T} b}{\sigma_{i}} v_{i}
$$

In Equation (14), if the filter factor defined as

$$
f_{i}= \begin{cases}1 & \sigma_{i} \geq \lambda_{i} \\ 0 & \sigma_{i}<\lambda_{i}\end{cases}
$$

Then the regularized solution is turn to the truncated SVD solution. Clearly, the key idea in Equation (14) is to modify the small singular values of matrix $\boldsymbol{A}$, and Equation (15) is use to decide from where the singular values should be modified. Here we propose a new method for modifying the singular values in a specific method, and its solution is noted as $x_{\text {reg- } k}$ which is called as the modified singular value (MSV) solution.

For solving ill-posed problems in GPS coordinate transformation by the Buras model, the characteristics of individual real model should be considered. That is, after the $i$ th, the true Fourier coefficients $\left|u_{i}^{T} b_{\text {exact }}\right|$ cannot decay faster than singular values, however, there is still some useful information exist in $\left|u_{i}^{T} b_{\text {exact }}\right|$, one of real example is presented in Figure 1. In Figure 1, singular values $\sigma_{i}$ is connected with circle dash line, true Fourier coefficients $\left|u_{i}^{T} b_{\text {exact }}\right|$ is connected with cross dash line, and total Fourier coefficients $\left|u_{i}^{T} \boldsymbol{b}\right|$ is connected with red circle solid line. We can see from $i=5$, error Fourier coefficients $\left|u_{i}^{T} b_{\text {noise }}\right|$ are greater than corresponding singular values $\sigma_{i}$, but the true Fourier coefficients $\left|u_{i}^{T} b_{\text {exact }}\right|$ are still significant. If we use TSVD method, the true Fourier coefficients from $i=5$ to $i=7$ will be discarded. Our new MSV method will try to use these discarded items for improving the solution.

As above, Tikhonov regularization is to modify those small singular values, so as to absorb discarded items by TSVD. However, this method also modifies large singular values, and thus influences the precision of coordinate transformation.

Our proposed MSV method is similar with Tikhonov regularization method, but only items with small singular values in Equation (14) will be modified and items with large singular values will be kept unchanged, that means $f=1$ in Equation (14).

\section{Estimation of Regularization Parameter}

The key question in regularization method is how to choose the regularization parameter, either the Tikhonov method's parameter $\lambda$ or the TSVD method's parameter $k$. Thus, estimation of regularization parameter plays a very importance role in solving ill-posed problems.

Perhaps the most convenient graphical tool for determining of regularization parameter is so-called $L$-curve which is a plot of regularization parameter solution (semi) norm e.g., the 2-(semi)norm $\|L x\|_{2}$, for all possible regularization parameters versus the corresponding residual norm e.g., $\|A x-b\|_{2}$. The L-curve clearly displays the compromise between minimization of these two quantities, 


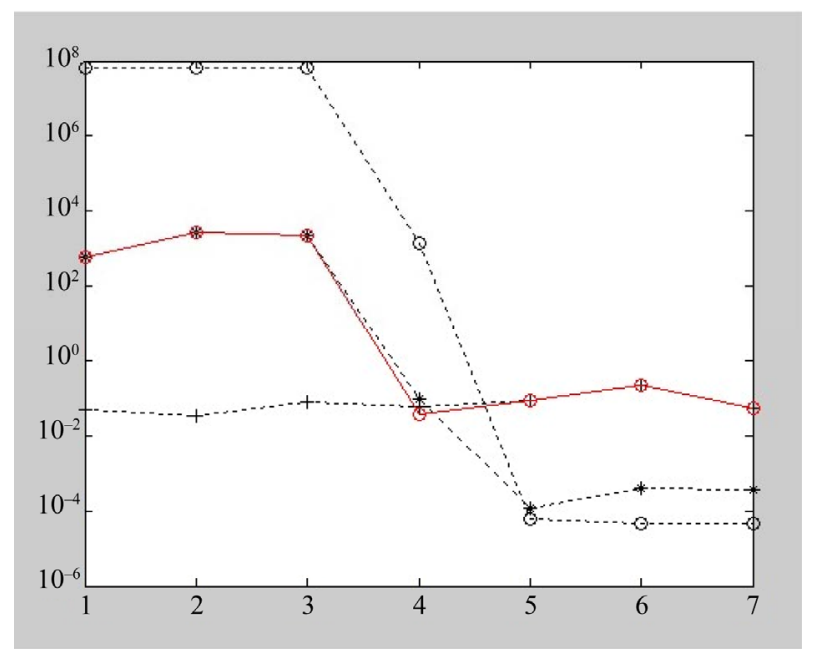

Figure 1. Compare singular values with Fourier coefficients. Singular values (circle with dash line), true Fourier coefficients (asterisks with dash line), error Fourier coefficients (crosses with dash line), and total Fourier coefficients (red circle with solid line) for coefficient matrix $A$ and observation vector $b$.

which is the main concerns of any regularization method. For some more details and analysis on $L$-curve, the reader may refer to Hansen [17], and also Hanke [18].

In geodetic problems, L-curve method has often induced oversmoothed solutions [19]. Minimizing the trace of mean squared error (MSE) method is also a powerful approach to estimate regularization parameter $\lambda$, Shen and $\mathrm{Li}$ [20] presented this method in GPS numerical treatment. In this method, minimizing the trace of mean squared error is required, the criterion as

$$
\min \operatorname{Trace}(\boldsymbol{M})
$$

where Trace(.) denotes the trace of the matrix, and $\boldsymbol{M}$ denotes the mean squared error matrix. From Shen and Li the second order derivative of Trace $(\boldsymbol{M})$ satisfies

$$
\frac{\mathrm{d}^{2} \operatorname{Trace}(\boldsymbol{M})}{\mathrm{d} \lambda^{2}}>0
$$

Thus the unique solution exists for Equation (16), and it can be solved by letting the first-order derivative of Trace $(\boldsymbol{M})$ to zero.

Another approach to estimate regularization parameter is through the condition number of coefficient matrix $\boldsymbol{A}$ [21], in this method a relation between the regularization parameter and the sensitivity of the regularized solution is investigated. As in Regińska

$$
\kappa(\boldsymbol{A})=\frac{\sigma_{\text {max }}(\boldsymbol{A})}{\sigma_{\text {min }}(\boldsymbol{A})}
$$

where $\kappa(A)$ shows the sensitivity of Equation (4) on data perturbations. In this method, through analysis of the optimal $\kappa^{o p t}$, the regularization parameter can be chosen as

$$
\lambda=\sigma_{\min }(\boldsymbol{A}) \sigma_{\max }(\boldsymbol{A})
$$

Many authors have discussed estimation of regularized parameter, and there are also some other methods, like Generalized Cross-Validation [22]. In fact, each method for estimating regularization parameter has different advantages and disadvantages. There is no unique method applicable to all ill-posed problems. Based on those early methods, we propose a new method to estimate regularization parameter in coordinate transformation.

Considering Equations (14) and (15), and the characteristics of coordinate transformation mentioned in the above, we concern how to modify the small singular values and from where they should be modified. The form of coefficient matrix $\boldsymbol{A}$ in coordinate transformation has been given by Equation (2), and the singular values, the true Fourier coefficients and the error Fourier coefficients of an example of matrix $\boldsymbol{A}$ are presented in Figure 1. As in Figure 1, the first three singular values are significant larger than zero and their corresponding total Fourier coefficients, moreover, the true Fourier coefficients are larger than their corresponding error Fourier coefficients, i.e., total Fourier coefficients are completely dominated by true Fourier coefficients, so we decide to keep these three singular values unmodified

$$
\sigma_{\text {modify, } i}=\sigma_{i} \quad i=1,2,3
$$

The singular value decays at the fourth, and the last three singular values are approximate to zero. The fourth total Fourier coefficient is less than the fourth singular value, thus ill-posed problems is not significant. However, Figure 1 shows the fourth error Fourier coefficient is almost equal to the fourth true Fourier coefficient, thus perturbations may influence the exact solution, so this singular value is modified as

$$
\sigma_{\bmod i f y, i}=\sigma_{i} \cdot \frac{\varepsilon}{\xi} \quad i=4
$$

where $\varepsilon, \xi$ denote error level and error adjustment coefficient, respectively (In our simulation examples, $\varepsilon=5 \mathrm{~cm}$ and choosing $\xi=4.53$ ).

The last three singular values are obviously smaller than the corresponding total Fourier coefficients. Moreover, the corresponding error Fourier coefficients are larger than the corresponding true Fourier coefficients respectively. Clearly, in this situation, the model is ill-posed, and the useful information is completely covered by perturbations. For keeping the useful information for coordinate transformation, the singular values are modified as

$$
\sigma_{\bmod i f y, i}=\sigma_{\max } \quad i=5,6,7
$$

So, we obtain "new" singular values using Equations (20)-(22) so as to the solution of our MSV method, it can be rewritten as 


$$
x_{\text {reg }-k}=\sum_{i=1}^{\operatorname{rank}(A)} \frac{u_{i}^{T} b}{\sigma_{\bmod i f y, i}} v_{i}
$$

\section{Numerical Experiments}

The data used in our experiments is the simulated coordinates of GPS stations distributed in 2000 square kilometers. We use five GPS points as control points, and apply "true" coordinate transformation parameters to get corresponding "true" coordinates in the local coordinate system. From initial investigations, we know that if points locate in the interior of a network composed by control points, we can get their corresponding coordinates with acceptable precision in a local coordinate system by coordinate transformation parameters even using the classical least squares method. Moreover, the results of coordinate transformation are also acceptable when coordinates of control points have smaller noise in both coordinate systems. So, the coordinates of points outside the region of the control network will be used to check the precision of coordinate transformation by different methods.

In our experiments, we give five centimeters perturbations to coordinates of control points in both systems. Firstly, we use the "true" transformation parameters to get some "true" coordinates outside the control network in both coordinate systems. Secondly, we transform coordinates of those outside points to the local system with different coordinate transformation parameters which obtained by different regularization methods, and compare each of them with their "true" coordinates.

Root mean square error (RMSE) of points by least squares method is presented in Figure 2. Here, we simulated coordinates of 100 points in exterior area with a white noise with zero means and standard deviations of 5

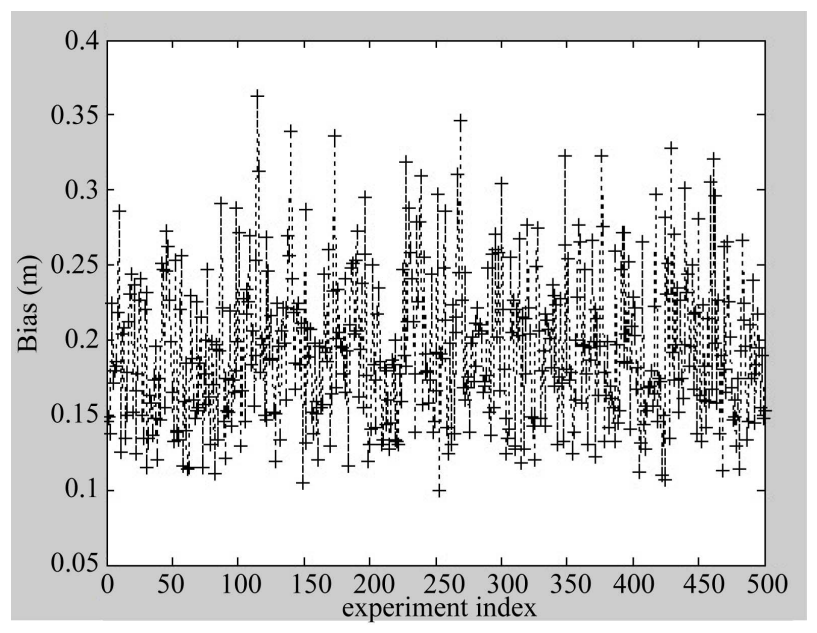

Figure 2. RMSE of transformed coordinates solving by least squares method. centimeters, and 500 coordinate transformation experiments have been repeated for obtaining mean of RMSE with statistical significance. Figure 2 shows that the mean of RMSE is clearly larger than the noise has been imposed in the coordinates, and the results have large oscillations.

The results solving by TSVD, Tikhonov regularization with L-curve, and MSE methods are presented in Figures 3(a)-(c) respectively. Clearly, the means of RMSE are smaller than the result in Figure 2, especially the mean value by MSE is approximate to the error which has been given before, and also has stronger robustness.

Figure 4 presents the results solving by our MSV method with the modified "new" singular values. Obviously, the results solving by MSV method have the smallest mean of RMSE and stronger robustness. Table 1 summarizes means of RMSE and their corresponding standard deviations of different methods.

The performance about two of those points in 500 tests by MSV method are presented in Figure 5. Clearly, The No. 68 point (red) is more seriously corrupted by noise than the No. 28 point (black). In order to present some good properties of our new method, the results of the No. 68 point by MSV, TSVD, and Tikhovon regularization with $L$-curve are drawn in Figures 6(a)-(c), respectively. Table 2 summarizes mean of RMSE and their corresponding standard deviations of No. 68 points through using different methods. Clearly, when the point has poor precision, our MSV method can balance the point precision and robustness well.

\section{Conclusions}

It is well known that regularization has been successfully applied to solve ill-posed problems by significantly improving the condition number of ill-condition matrix. A very large condition number is usually caused by the small singular values of matrix, so we propose a new me-

Table 1. Mean of RMSE and standard deviations.

\begin{tabular}{ccc}
\hline & Mean of RMSE $(\mathrm{m})$ & Standard Deviations \\
\hline LS & 0.1930 & 0.04989 \\
TSVD & 0.1408 & 0.04648 \\
L-curve & 0.1347 & 0.03743 \\
MES & 0.1351 & 0.02858 \\
$M S V$ & 0.1116 & 0.03384 \\
\hline
\end{tabular}

Table 2. Mean of RMSE and standard deviations of No. 68 point.

\begin{tabular}{ccc}
\hline & Mean of RMSE $(\mathrm{m})$ & Standard Deviations \\
\hline$M S V$ & 0.1561 & 0.08363 \\
$T S V D$ & 0.2076 & 0.1034 \\
L-curve & 0.1634 & 0.0910 \\
\hline
\end{tabular}




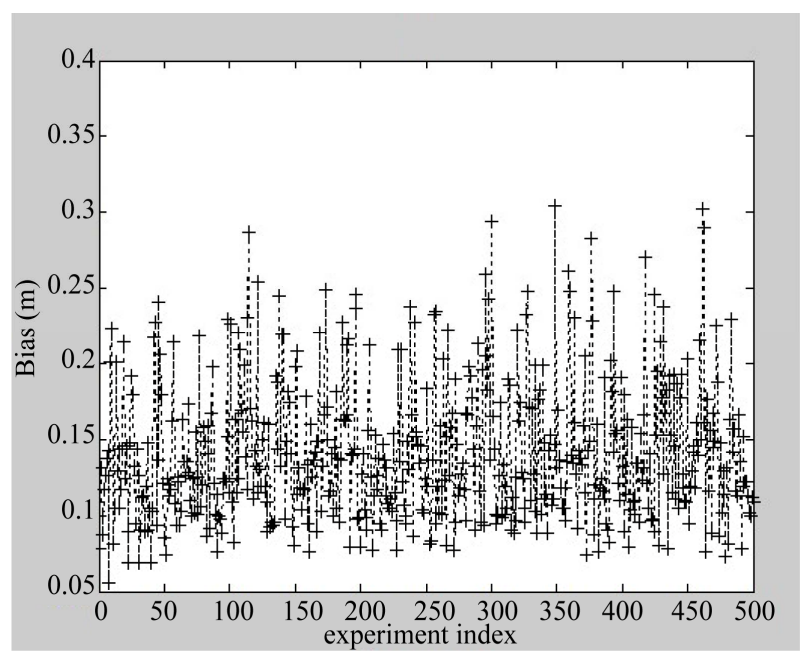

(a)

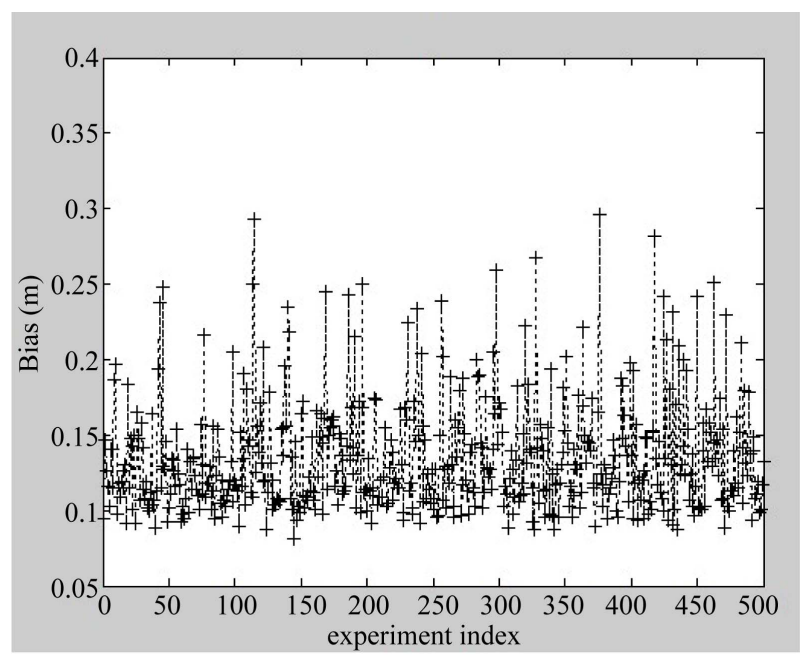

(b)

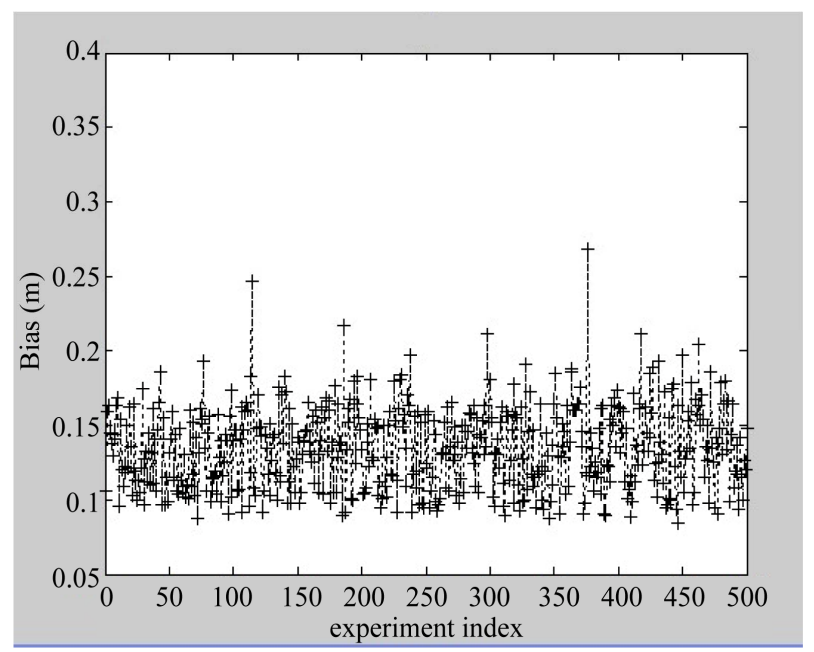

(c)

Figure 3. RMSE of transformed coordinates solving by TSVD (a); Tikhonov regularization with $L$-curve (b); and MSE (c) methods.

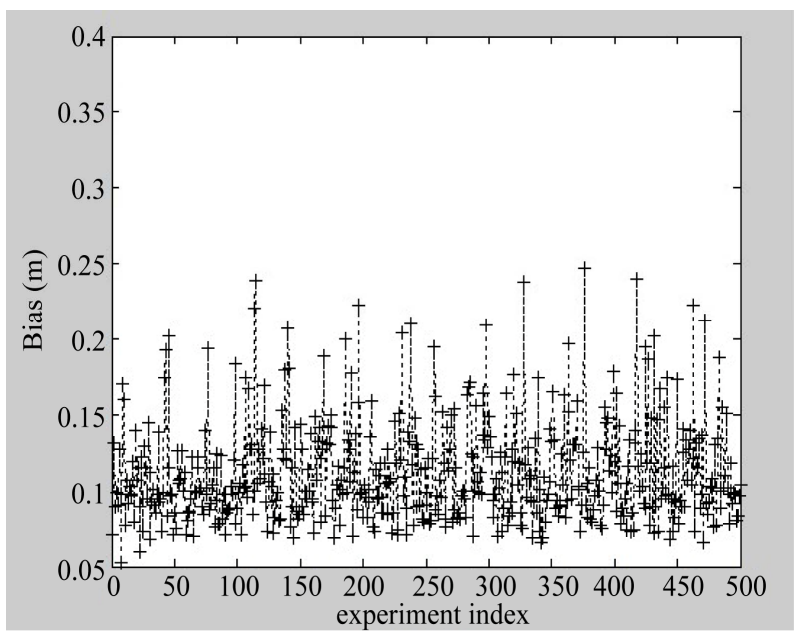

Figure 4. RMSE of transformed coordinates solving by MSV with "new" singular values by new method.

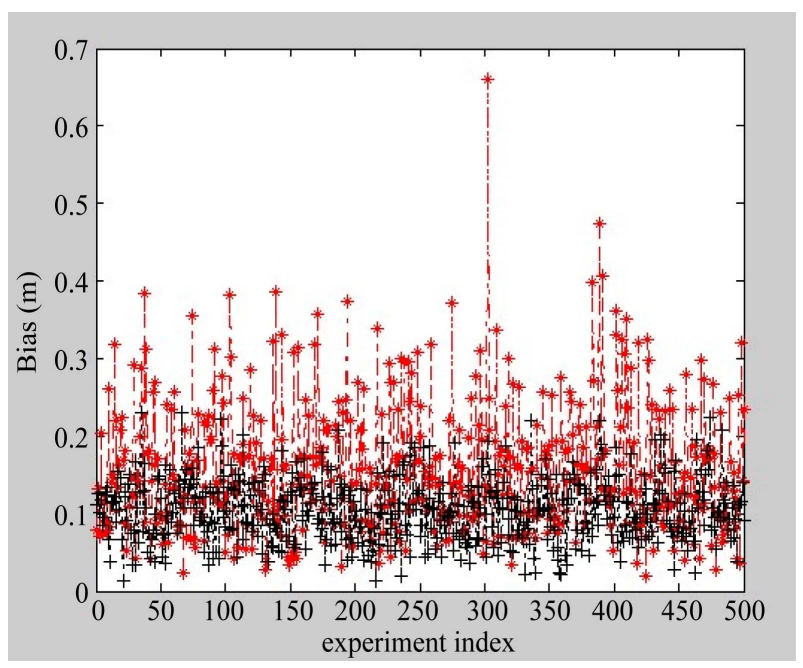

Figure 5. Point error of the No. 28 (black) and No. 68 (red) solving by new method.

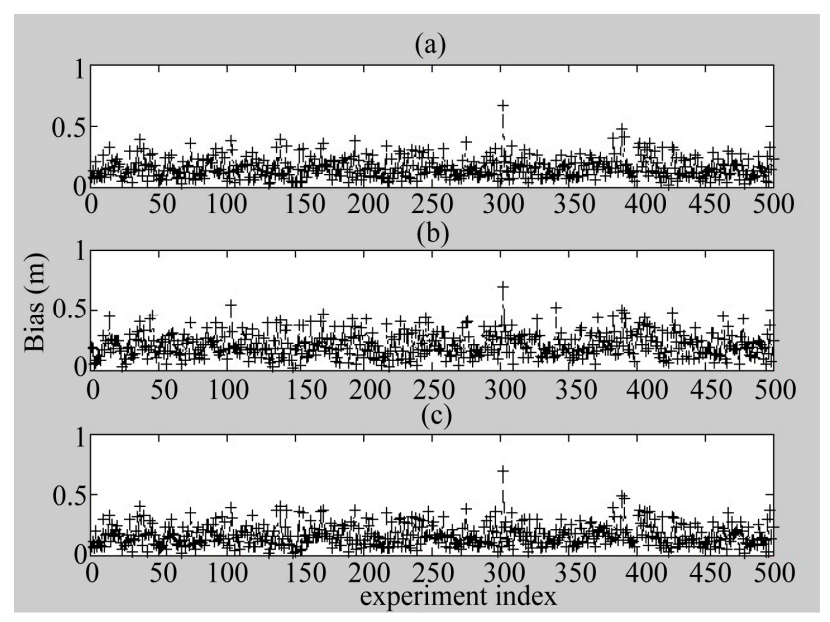

Figure 6. Point error of the No.68 solving by new method (a); TSVD (b); and Tikhonov regularization with $L$-curve (c). 
thod for solving ill-posed problems in coordinate transformation through modifying small singular values. The numerical experiments have demons-trated that the new method can solve these kinds of ill-posed problems, and gain an applicable precision, moreover, perform stronger robustness. In practical coordinate transformation problems, we do not know the true and error Fourier coefficients, the total Fourier coefficients can be used to modify the singular values so as to implement the MSV method.

\section{Acknowledgements}

This paper is sponsored by the Natural Science Foundation of China (No. 41074019) and International cooperation project of Chinese ministry of Science (No. 2010DFB20190).

\section{REFERENCES}

[1] E. W. Grafarend, F. Krumm and F. Okeke, "Curvilinear Geodetic Datum Transformations," Z Vermessungswesen, Vol. 120, No. 4, 1995, pp. 334-350.

[2] P. Vanicek and R. R. Steeves, "Transformation of Coordinates between Two Horizontal Geodetic Datum," Journal of Geodesy, Vol. 70, No. 11, 1996, pp. 740-745.

[3] P. Vanicek, P. Novak and R. Craymerm, "On the Correct Determination of Transformation Parameters of Horizontal Geodetic Datum," Geomatica, Vol. 56, No. 4, 2002, pp. 329-340.

[4] Y. Yang, "Robust Estimation of Geodetic Datum Transformation," Journal of Geodesy, Vol. 73, No. 9, 1999, pp. 268-274. doi:10.1007/s001900050243

[5] E. W. Grafarend and L. J. Awange, "Nonlinear Analysis of the Transformational Datum Transformation," Journal of Geodesy, Vol. 77, 2003, pp. 66-76. doi:10.1007/s00190-002-0299-9

[6] A. N. Tikhonov, "Regularization of Ill-Posed Problems," Doklady Akademi Nauk, Vol. 151, No. 1, 1963, pp. 4952.

[7] A. N. Tikhonov, "Solution of Incurrectly Formulated Problems and the Regularization Method," Doklady Akademi Nauk, Vol. 151, No. 3, 1963, pp. 501-504.

[8] G. H. Golub and C. Reinsch, "Singular Value Decomposition and Least Squares Solutions," Numerical Mathematics, Vol. 14, 1970, pp. 403-420. doi:10.1007/BF02163027
[9] P. C. Hansen, "The Truncated SVD as a Method for Regularization,” BIT, Vol. 27, 1987, pp. 534-553. doi:10.1007/BF01937276

[10] P. L. Xu and R. Rummel, "Generalized Ridge Regression with Applications in Determination of Potential Fields," Manuscripta Geodaetica, Vol. 20, No. 1, 1994, pp. 8-20.

[11] P. L. Xu, Y. Fukuda and Y. Liu, "Multiple Parameter Regularization: Numerical Solution and Application to the Determination of Geopotential from Precise Satellite Orbits," Journal of Geodesy, Vol. 80, No. 1, 2006, pp. 17-27. doi:10.1007/s00190-006-0025-0

[12] G. H. Golub and C. F. Van Loan, "Matrix Computation," 3rd Edition, The Johns Hopkins University Press, Baltimore, 1996.

[13] P. C. Hansen, "Rank-Deficient and Discrete Ill-Posed Problems," SIAM, Philadelphia, 1998. doi:10.1137/1.9780898719697

[14] P. Tarantola, "Inverse Problem Theory," SIAM, Philadelphia, 2005.

[15] P. C. Hansen, "The Discrete Picard Condition for Discrete Ill-Posed Problems," BIT, Vol. 30, No. 4, 1990, pp. 658-672. doi:10.1007/BF01933214

[16] P. C. Hansen, "Truncated SVD Solutions to Discrete IllPosed Problems with Ill-Determined Numerical Rank," Journal on Scientific and Statistical Computing, Vol. 11, 1990, pp. 503-518. doi:10.1137/0911028

[17] P. C. Hansen, "Analysis of Discrete Ill-Posed Problems by Means of the L-Curve," SIAM Review, Vol. 34, No. 4, 1992, pp. 561-580. doi:10.1137/1034115

[18] M. Hanke, "Limitations of the L-Curve Method in IllPosed Problems,” BIT, Vol. 36, No. 2, 1996, pp. 287-301. doi:10.1007/BF01731984

[19] P. L. Xu, "Truncated SVD Methods for Discrete Linear Ill-Posed Problems," Geophysical Journal International, Vol. 135, No. 2, 1998, pp. 505-514. doi:10.1046/j.1365-246X.1998.00652.x

[20] Y. Z. Shen and B. F. Li, "Regularized Solution to Fast GPS Ambiguity Resolution," Journal of Surveying Engineering, Vol. 133, No. 4, 2007, pp. 168-172. doi:10.1061/(ASCE)0733-9453(2007)133:4(168)

[21] T. Regińska, "Regularization of Discrete Ill-Posed Problems," BIT, Vol. 44, No. 3, 2004, pp. 119-133.

[22] G. H. Golub, M. T. Heath and G. Wahba, "Generalized Cross-Validation as a Method for Choosing a Good Ridge Parameter," Technometrics, Vol. 21, No. 2, 1979, pp. 215 223. doi: $10.2307 / 1268518$ 